\title{
Improved Harris Feature Point Set for Orientation-Sensitive Urban-Area Detection in Aerial Images
}

\author{
Andrea Kovács, Student Member, IEEE, and Tamás Szirányi, Senior Member, IEEE
}

\begin{abstract}
This letter addresses the automatic detection of urban area in remotely sensed images. As manual administration is time consuming and unfeasible, researchers have to focus on automated processing techniques, which can handle various image characteristics and huge amount of data. The applied method extracts feature points in the first step, which is followed by the construction of a voting map to represent urban areas. Finally, an adaptive decision making is performed to find urban areas. This letter presents methodological contributions in two key issues to the algorithm: 1) An automatically extracted Harris-based feature point set is introduced for the first step, which is able to represent urban areas more precisely. 2) An improved orientation-sensitive voting technique is proposed, exploiting the orientation information calculated in the local neighborhood of points. Evaluation results show that the proposed contributions increase the detection accuracy of urban areas.
\end{abstract}

Index Terms-Aerial images, modified Harris detector, orientation sensitivity, spatial voting, urban-area detection.

\section{INTRODUCTION}

$\mathbf{T}$ HE automatic detection of urban areas in optical aerial images means a great support in urban development analysis, map updating, and disaster management, and also helps municipalities in long-term residential area planning. Unfortunately, the images may cover large areas, and they can be taken in altering weather and illumination conditions, causing very different image features. Moreover, as urban areas are usually dynamically changing, continuous administration is required to have up-to-date information. Since manual administration is time consuming and unfeasible, researchers have to focus on automated processing techniques, which can handle various image characteristics and huge amount of data.

A wide range of automatic urban (a.k.a. built-in) area detection techniques have been introduced in recent years. The first group of such methods needs specific training data to detect urban areas, such as [1], using a differential morphological profile to record structural image information then applied feature extraction and neural network for classifying the fea-

Manuscript received May 25, 2012; revised September 14, 2012; accepted October 8, 2012. This work was supported by the Hungarian Scientific Research Fund under Grant 76159 and Grant 80352.

The authors are with the Distributed Events Analysis Research Laboratory, Computer and Automation Research Institute, Hungarian Academy of Sciences, 1111 Budapest, Hungary, and also with the Faculty of Information Technology, Pazmany Peter Catholic University, 1083 Budapest, Hungary (e-mail: andrea.kovacs@sztaki.mta.hu; sziranyi@ sztaki.mta.hu).

Color versions of one or more of the figures in this paper are available online at http://ieeexplore.ieee.org.

Digital Object Identifier 10.1109/LGRS.2012.2224315 tures, and [2], presenting a multiple conditional random-field ensemble model to incorporate multiple features and learn their contextual information.

Our letter followed another methodology, i.e., to construct a direct method that does not need any training data for urban-area extraction. Martinez-Fonte et al. [3] showed that corner detectors (Harris [4] and SUSAN [5]) are efficient tools for distinguishing different types of structures (manmade versus natural structures) present in the image. Since then, other works also applied interest point detectors for urbanarea detection; [6] used scale-invariant feature transform (SIFT) integrated with graph theory. The results were promising, but the computational complexity and time were quite significant. To reduce computational requirements, the same authors introduced a novel technique using Gabor feature points and spatial voting [7].

In this letter, we first introduce a new automatically extracted feature point set, which is called Modified Harris for Edges and Corners (MHEC) for urban-area detection, which was applied for effective object contour detection in our earlier work [8]. As the density of extracted feature points is higher in the residential areas [3], building a probability map based on this feature point set can identify urban areas. After having a local feature point set, we apply the voting matrix strategy of [7] to get a probability map of the urban area. To improve the accuracy of this step, we also propose a novel orientationsensitive technique for constructing the voting matrix. Finally, urban areas are calculated by a decision making step. Fig. 1 shows the main algorithmic steps of the method. In the experimental part, we will show that the introduced method is able to outperform other interest point detectors; moreover, the proposed orientation-sensitive voting matrix is also able to improve the performance of the previously introduced Gaborbased algorithm [7].

\section{MODIFIED HARRIS FOR EDGES AND CORNERS}

We introduce a novel MHEC feature point set for urbanarea detection, which is based on the Harris corner detector [4] but adopts a modified characteristic function. The advantage of the improved detector is that it is automatic and it is able to recognize not just corners but edges as well. Therefore, it gives an efficient tool for characterizing contour-rich regions, such as urban areas. The improved characteristic function was also successfully applied earlier for object contour recognition with parametric active contour algorithms [8].

By considering the behavior of the Harris matrix and its eigenvalues (denoted by $\lambda_{1}$ and $\lambda_{2}$ ), we proposed the following 


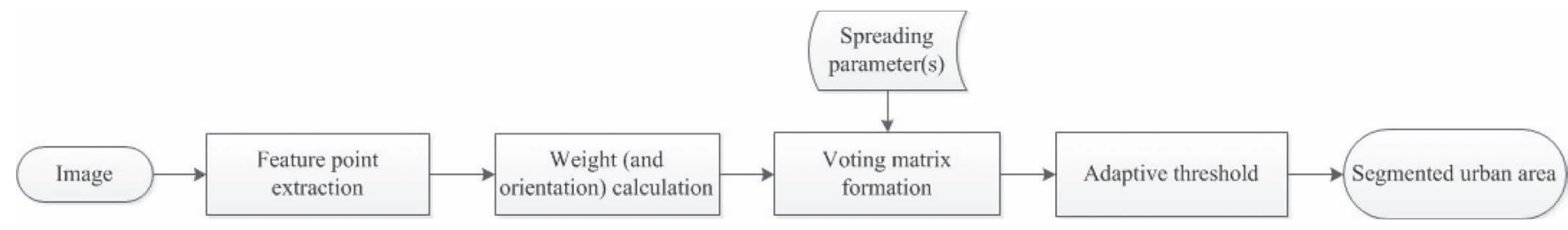

Fig. 1. Simplified diagram of the workflow.

modification of the $R$ characteristic function in our earlier work [8]:

$$
R_{\text {mod }}=\max \left(\lambda_{1}, \lambda_{2}\right)
$$

As our aim is to emphasize edge and corner regions simultaneously, we can exploit the fact that they both have one large eigenvalue. Therefore, the suggested $R_{\text {mod }}$ value is able to separate homogeneous and nonhomogeneous regions of the image.

Feature points are calculated as the local maxima of $R_{\text {mod }}$. A pixel $p_{i}=\left(x_{i}, y_{i}\right)$ is the element of the $P$ feature point set, if it has the largest $R_{\text {mod }}\left(p_{i}\right)$ value compared with its neighbors in a surrounding $b_{i}=\left\{\left[x_{i}-1, x_{i}+1\right] \times\left[y_{i}-1, y_{i}+1\right]\right\}$ window and its $R_{\bmod }\left(p_{i}\right)$ value exceeds a given $T_{\max }$ threshold, i.e.,

$P=\left\{p_{i}: R_{\text {mod }}\left(p_{i}\right)>T_{\max } \operatorname{AND} p_{i}=\arg \max _{r \in b_{i}} R_{\bmod }(r)\right\}$.

Here, the $T_{\max }$ threshold is calculated by Otsu's method [9] for each image adaptively; therefore, it can handle different image characteristics.

By investigating the smaller eigenvalue $\min \left(\lambda_{1}, \lambda_{2}\right)$, edge and corner points can be separated. If $\min \left(\lambda_{1}, \lambda_{2}\right)$ is over an adaptive $T_{\min }$ threshold (also calculated by Otsu's method), then the point is a corner point candidate; otherwise, it is an edge point. The separation of edge and corner points will be used in Section III-B.

The proposed feature point detector is completely automatic and does not need any parameter tuning, unlike the referred Gabor mechanism [7]. The method is also not sensitive to the $g$ scale parameter of the Gaussian smoothing used in the Harris corner detection; we picked the $g=0.5$ value for every test case, regardless of resolution or any other image feature. Therefore, we did not present it in Fig. 1 as the input parameter. Additionally, the aforementioned $T_{\min }$ and $T_{\max }$ thresholds are defined with Otsu's adaptive method.

In the next step (see Fig. 1), we calculate a weight value (presented in [7]) for each feature point that represents the strength of the point's neighborhood. Following [7], we binarize the $R_{\text {mod }}$ map with the adaptive $T_{\max }$ threshold to get a $B$ binary map. In $B$, pixels of $R_{\bmod }$ over $T_{\max }$ get 1 ; others get 0 value. By definition, every member of the $P$ feature point set will have a value of 1 [see (2)]. Let $w_{i}$ denote the weight of the $p_{i}$ point, which is calculated as the natural logarithm of the number of pixels connected to $p_{i}$ in the $B$ binary map. Two pixels are connected in $B$ if both pixels are 1, and a path of pixels with a value of 1 exists between them. If the number of connected pixels is less than 10 (defined based on the recommendation of [7]), the point is classified as nonsignificant and eliminated from the feature point set. Points in larger sections, like roads, may have higher weights than others, resulting in an unbalanced weighting function;
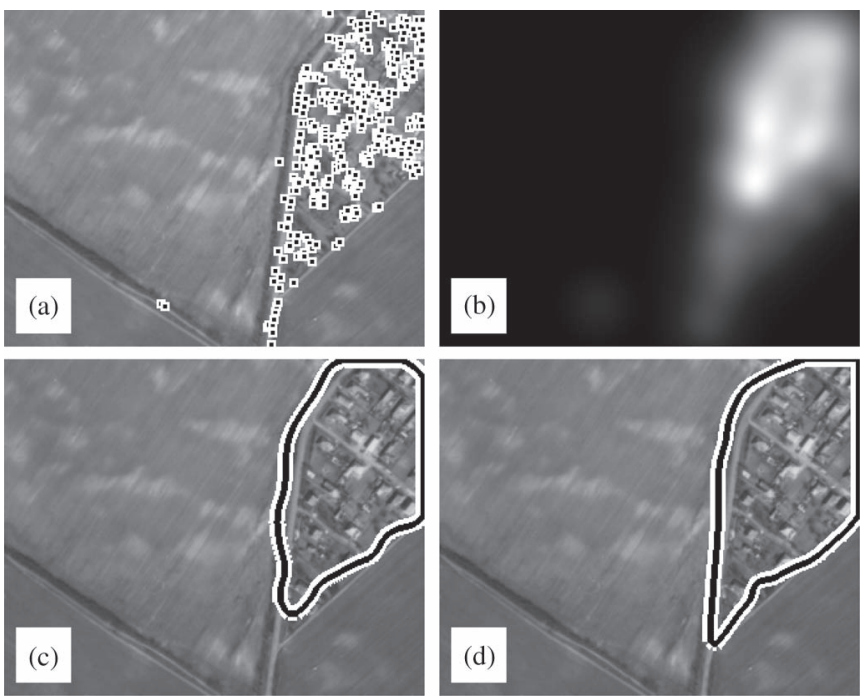

Fig. 2. Steps of the urban-area extraction for the Szada 1 image with the proposed MHEC feature point set. (a) Extracted feature point set. (b) Voting matrix of the referred nonoriented process [7]. (c) Detected urban area applying the nonoriented process. (d) Detected urban area applying the improved orientation-sensitive process.

therefore, the reason for applying the natural logarithm function on the calculated weights is to get steady $w_{i}$ values.

The modified Harris point set for the $\mathrm{Szada}_{1}$ image is shown in Fig. 2(a). The modified (extended) point set has members in edge regions (like roads) as well, and points are densely located in the built-in area. Only a few points are situated in the nonurban area.

\section{Orientation-SEnsitive Urban-AREA EXtraction}

\section{A. Orientation-Sensitive Voting Matrix Formation}

Sirmaçek and Ünsalan [7] assumes that, around Gabor feature points [10], an urban area can be found with a high probability. Thus, it defines a Gaussian domain around each $p_{i}$ point with $\sigma_{i}$ standard deviation, where the highest vote is the point, and according to the spatial distance, the vote is decreasing. $\sigma_{i}$ controls the spatial effect of a point; if it is higher, a point has wider impact. The significance of a point is proportional to its weight; thus, $\sigma_{i}$ is estimated by $w_{i}$. The $V$ voting matrix for the $(x, y)$ pixel is defined as

$$
V(x, y)=\sum_{i=1}^{K} \frac{1}{2 \pi \sigma_{i}^{2}} e^{-\frac{\left(x-x_{i}\right)^{2}+\left(y-y_{i}\right)^{2}}{2 \sigma_{i}^{2}}}
$$

where $K$ is the total number of feature points, and $\sigma_{i}=5 \times w_{i}$. Fig. 2(b) shows the generated $V$ map for the $\mathrm{Szada}_{1}$ image. Finally, the urban area is defined by an adaptive decision making step. Otsu's thresholding technique [9] is applied on $V$ to distinguish the urban area from the background. The detection result is shown in Fig. 2(c). 

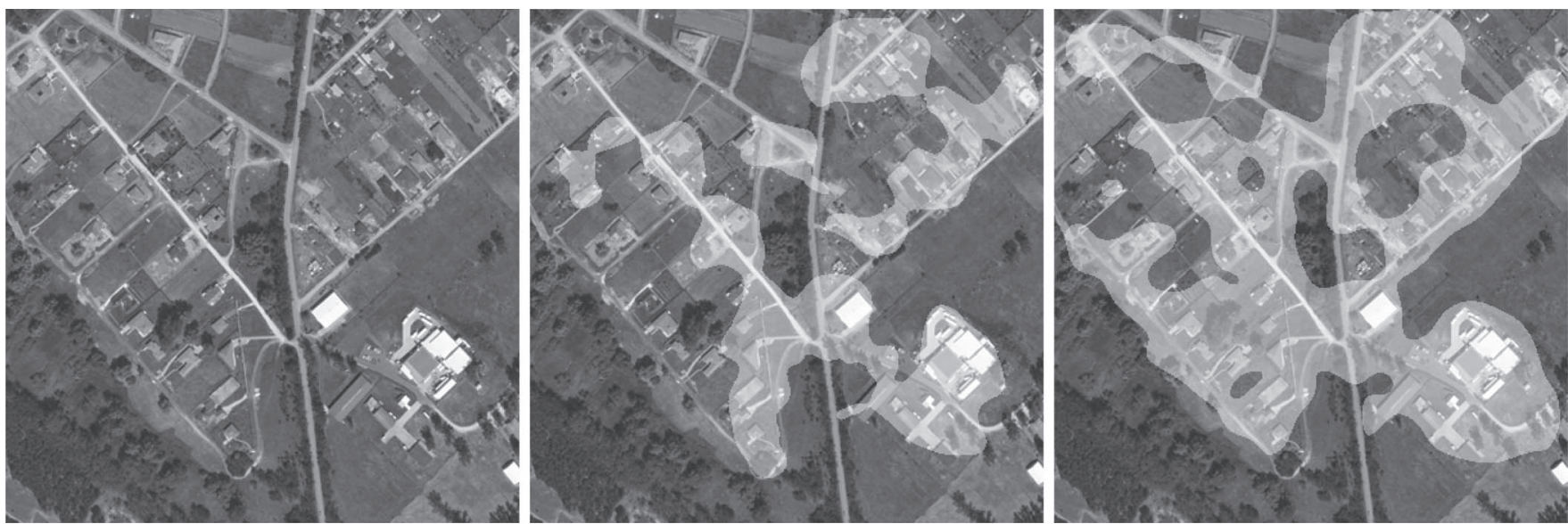

Fig. 3. Detection results for the Szada5 image with different voting matrix formation techniques. (Left) Original image. (Middle) Result of the original nonoriented method [7] ( $F$-measure value: 0.548$)$. (Right) Result of the proposed orientation-sensitive method ( $F$-measure value: 0.717$)$.

The disadvantage of the method is that it is not able to detect straight lines accurately because of the circular Gaussian representation. If a straight urban-area border (like a road segment or a row of houses) is represented by sparse feature points, then the voting matrix will have high values only in the neighborhood of the points; therefore, the detection will result in a less accurate wavy urban-area border. Moreover, high curvature boundaries are also inaccurately detected.

To compensate these drawbacks, we introduce the orientation-sensitive extension of the voting matrix. Our idea is to use the direction information of Gabor filters [10] when constructing the voting matrix. Therefore, we propose to apply the 2-D elliptical Gaussian function at the feature point $\left(x_{i}, y_{i}\right)$, which can model orientation:

$$
\begin{aligned}
& G_{i}(x, y)=A_{i} \cdot E_{i}(x, y) \\
& E_{i}(x, y)=e^{-\left(a_{i}\left(x-x_{i}\right)^{2}+2 b_{i}\left(x-x_{i}\right)\left(y-y_{i}\right)+c_{i}\left(y-y_{i}\right)^{2}\right)}
\end{aligned}
$$

where the calculation of $A_{i}$ is given in (7), and $a_{i}, b_{i}$, and $c_{i}$ are calculated in the following way:

$$
\begin{aligned}
a_{i} & =\frac{\cos ^{2} \theta}{2 \sigma_{i, x}^{2}}+\frac{\sin ^{2} \theta}{2 \sigma_{i, y}^{2}} \\
b_{i} & =-\frac{\sin 2 \theta}{4 \sigma_{i, x}^{2}}+\frac{\sin 2 \theta}{4 \sigma_{i, y}^{2}} \\
c_{i} & =\frac{\sin ^{2} \theta}{2 \sigma_{i, x}^{2}}+\frac{\cos ^{2} \theta}{2 \sigma_{i, y}^{2}}
\end{aligned}
$$

where $\sigma_{i, x}$ and $\sigma_{i, y}$ are the spreads of the blob in the $x$ - and $y$ directions, and $\theta$ is the orientation for the $\left(x_{i}, y_{i}\right)$ feature point.

This interpretation represents a 2-D elliptical Gaussian function, which is rotated by a clockwise angle $\theta$. If we define $\theta$ as the orientation of the Gabor filter, which extracted the feature point, then we can exploit the additional direction information in the voting matrix formulation by expanding a point's impact in the main $\theta$-direction.

Regarding this consideration, we define the following oriented voting matrix:

$$
V_{\theta}(x, y)=\sum_{i=1}^{K} G_{i}(x, y)=\sum_{i=1}^{K} \frac{1}{2 \pi\left(\sigma_{i, x}^{2}+\sigma_{i, y}^{2}\right)} \cdot E_{i}(x, y) .
$$

We set the $\sigma_{i, y} / \sigma_{i, x}=2$ ratio to avoid too moderate voting in the minor axis direction, resulting in false negative hits in the detection step.

The effect of the orientation sensitivity is shown in Fig. 3, where the middle image shows the result of the original nonoriented method for Gabor points [7], while the right image shows the result of the improved orientation-sensitive technique $\left(\sigma_{i, x}=3 \times w_{i}\right.$ and $\left.\sigma_{i, y}=6 \times w_{i}\right)$. To quantitatively compare the results, the gained $F$-measure scores are also presented. (For further evaluation details see Section IV.)

\section{B. Orientation-Sensitive Voting Matrix Formation for the Novel Feature Point Set}

In case of a Gabor feature point set, defining a point's orientation does not need any extra computation; therefore, it is favorable to exploit the additional information. However, there may be other interest point detectors where the orientation has to be calculated in a distinct step. Moreover, in some cases, no such direction can be interpreted for points without further consideration. For example, when using the Harris corner detector, more than one main direction can be assigned to a point, which may pose many interpretation questions. (How is it to decide if a direction is significant? How many significant directions have to be calculated?)

In our case, the feature point set proposed in Section II will consist of both edge and corner points. Thus, calculating multiple directions featuring corner points is problematic. Meanwhile, edge points represent a main orientation, which can be defined, for example, by analyzing the small neighborhood, as in [11]. Therefore, we propose to apply a mixture of voting matrices $\left(V\right.$ and $\left.V_{\theta}\right)$. After dividing the feature point set into two subsets, i.e., corner and edge points (as described in Section II), the original circular Gaussian field $[V$ in (3)] is calculated for corner points, and the improved orientationsensitive elliptical field $\left[V_{\theta}\right.$ in (7)] is calculated for edge points. The final voting matrix is then given as the pixelwise sum of the two precalculated matrices. Fig. 2(d) shows the result of the joint detection technique for the proposed MHEC point set.

\section{EXPERIMENTS}

For testing the performance of the proposed feature point set and the improved orientation-sensitive voting matrix, we 
used the Szada data set provided by the Hungarian Institute of Geodesy, Cartography, and Remote Sensing (FÖMI; earlier used in [15]). The Szada test set contains 11 aerial images taken by FÖMI in 1984, 2000, and 2005, showing the Szada village and its close proximity. Our aerial images have a different spatial resolution, i.e., 1.5 or $0.5 \mathrm{~m} / \mathrm{pixel}$; images are either grayscale or colored. The size of the images is varying from $320 \times 256$ to $996 \times 558$ pixels. Therefore, the data set contains diverse images to represent different urban region characteristics and to show the robustness of the proposed techniques.

In the first part of the evaluation process we investigate the efficiency of different interest point detectors for urban-area detection with the original nonoriented voting matrix technique. In the second part, we test the performance of the improved orientation-sensitive voting matrix for selected feature point detectors.

For comparing the results, we use $P$ precision, $R$ recall, and $F$-measure values, i.e.,

$$
P=\frac{\mathrm{TP}}{\mathrm{TP}+\mathrm{FP}} \quad R=\frac{\mathrm{TP}}{\mathrm{TP}+\mathrm{FN}} \quad F=2 \cdot \frac{P \cdot R}{P+R}
$$

where TP, FP, and FN denote the number of true positive, false positive, and false negative detected pixels, respectively.

When defining an urban area, various interpretations might be considered.

- Are roads and gardens parts of the urban area?

- Should buildings be separately or collectively marked?

To handle these subjective cases, we asked three individuals to label the urban area manually. If a pixel was labeled as urban by at least two persons, then it was treated as a part of a built-in area in the ground-truth-based evaluation process.

\section{A. Tests on Different Interest Point Detectors}

To evaluate the performance of our proposed feature point detector, we tested the original voting matrix technique for different point detectors. Since there exist a large number of point detection methods [16], we picked detectors so as to cover a wide range of detection techniques. Harris [4] and SUSAN [5] were proposed in [3] for extraction of manmade structures; previous works of Sirmaçek and Ünsalan used Lowe's SIFT [14] method in [6], Gabor filtering [10] in [7], and Features from Accelerated Segment Test (FAST) [12] and Lindeberg's blob detector Laplacian of Gaussian (LoG) [13] in [17]. Thus, we chose these detectors for evaluation and comparison.

Table I shows the calculated average $F$-measure values [see (8)] for different interest point detection methods. Our proposed MHEC method outperforms the other detectors and achieves more than 0.8 for a mean $F$-measure value.

The computation time was calculated for the MHEC feature point extraction step on the Szada 1 test image (with $320 \times$ 256 size), and altogether, $0.6 \mathrm{~s}$ has been achieved on a personal computer with an Intel Core i7 2.67-GHz central processing unit with 4-GB random access memory and MATLAB R2011b. This is a fairly good time compared with [7], where Gabor filtering and local feature point extraction steps needed approximately $1.5 \mathrm{~s}$ for an image with a similar size $(235 \times 265)$.
TABLE I

Average $F$-Measure Score (MeAn \pm Standard Deviation) For the Evaluated Feature Point Detector Methods FOR THE SZADA DATA SET

\begin{tabular}{|c|c|}
\hline Feature point detector & Average F-measure Score \\
\hline MHEC (proposed) & $\mathbf{0 . 8 0 1} \pm \mathbf{0 . 0 4 4}$ \\
\hline FAST [12] & $0.792 \pm 0.072$ \\
\hline SUSAN [5] & $0.778 \pm 0.084$ \\
\hline LoG [13] & $0.750 \pm 0.061$ \\
\hline SIFT [14] & $0.670 \pm 0.093$ \\
\hline Harris [4] & $0.667 \pm 0.154$ \\
\hline Gabor [7] & $0.646 \pm 0.124$ \\
\hline
\end{tabular}

\section{B. Tests on Orientation Sensitivity}

To test the orientation sensitivity, we first chose interest point detectors, where the orientation is reasonable. As mentioned in Section III-B, this additional information cannot be interpreted for every detector (like Harris detector or blob detectors) and sometimes needs more consideration like MHEC).

For the originally used Gabor points, orientation information for a point is given when determining the direction of the Gabor filter. Therefore, exploiting orientation information does not need any additional computation. Furthermore, detectors based on edge-enhancing techniques extract edge points as well, which can be thus used for orientation-sensitive urbanarea detection with further considerations.

In our evaluation step, we picked the proposed MHEC method and the SUSAN detector to show the performance of the orientation-sensitive voting matrix. In both cases, we used the mixture of nonoriented and oriented voting matrix techniques (see Section III-B), as edge and corner points can be separated. The separation procedure for the MHEC method was described in Section II. For the SUSAN method, the main principle can be used for both edge and corner detection purposes. Depending on the value of the geometric threshold, the algorithm is able to find edge or corner points (see [5] for further details); therefore, it can be used to distinguish edges and corners.

Fig. 4 shows the comparison of the performance for the three selected algorithms. The light bar is the original $F$-measure performance value achieved by the referred nonoriented method, while the dark one is the result achieved by the improved orientation-sensitive voting technique.

Spreading the parameter values of the orientation-sensitive voting matrix, $\sigma_{i, x}$ and $\sigma_{i, y}$ were selected based on the number of detected points and on the distribution of weight values. While the $\sigma_{i, y} / \sigma_{i, x}$ ratio handles the orientation sensitivity, i.e., the shape of the effect (depending on the number of points), $\sigma_{i, x}$ and $\sigma_{i, y}$ values are responsible for the coverage of a point (depending on the variance of weight values).

In case of Gabor points, we applied $\sigma_{i, x}=3 \times w_{i}$ and $\sigma_{i, y}=$ $6 \times w_{i}$ values. As this detector gives the highest number of points and the weight values have a large variance, orientation and saliency are represented by effectively by the points.

In case of SUSAN, the oriented $\sigma_{i, x}, \sigma_{i, y}$ (for edge points) and nonoriented $\sigma_{i}$ (for corner points) values were manually tuned with the following restrictions: $\sigma_{i, y} / \sigma_{i, x}=2$ ratio 


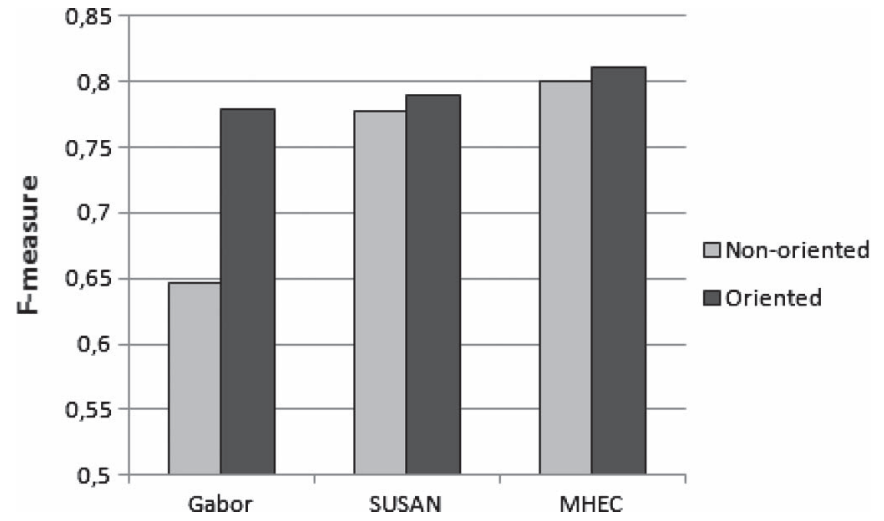

Fig. 4. Detection results based for different voting matrix techniques. (Left) Original nonoriented method [7]. (Right) Proposed orientation-sensitive method.

and $\sigma_{i, x}<\sigma_{i}<\sigma_{i, y}$ (a typical spreading parameter setting was $\sigma_{i, x}=8 \times w_{i}, \sigma_{i, y}=16 \times w_{i}$, and $\sigma_{i}=14 \times w_{i}$ used for $\mathrm{Szada}_{1}$ in Fig. 2). These considerations were empirically defined to balance the effect of corner and edge points. The variance of the weights was much lower than in the case of the Gabor detector; therefore, we selected larger multipliers in the case of $\sigma_{i, x}$ and $\sigma_{i, y}$ to exaggerate the saliency effect. The exact parameter values also depended on the resolution of the image; the higher the resolution, the wider spatial effect for a point has to be selected. Therefore, in the case of images with $0.5 \mathrm{~m} /$ pixel resolution, a typical parameter setting $\sigma_{i, x}=12 \times$ $w_{i}, \sigma_{i, y}=24 \times w_{i}$, and $\sigma_{i}=20 \times w_{i}$ was applied.

In the case of MHEC, the behavior of the detector was similar to the Gabor case, but the number of points was lower. Therefore, we set the $\sigma_{i, y} / \sigma_{i, x}$ ratio higher; to represent the orientation sensitivity more efficiently, we applied $\sigma_{i, x}=$ $2 \times w_{i}, \sigma_{i, y}=6 \times w_{i}$, and $\sigma_{i}=3 \times w_{i}$ for lower resolution, and $\sigma_{i, x}=2 \times w_{i}, \sigma_{i, y}=8 \times w_{i}$ and $\sigma_{i}=6 \times w_{i}$ for higher resolution. As the variance of the weight values was high, the multipliers of the $w_{i}$ weight values are smaller than in the case of SUSAN.

According to Fig. 4, the orientation-sensitive representation was able to improve the performance of urban-area detection. The improvement was the most significant for Gabor points, where no additional calculation was needed. Moreover, it caused a slight increase in the performances of SUSAN and MHEC as well.

\section{CONCLUSION}

We have proposed a feature point set based on the modification of the Harris corner detection method for urban-area extraction in aerial images. The point extraction method is fully automatic and is able to emphasize edge and corner points in the image equally; therefore, it serves as a suitable base for the construction of the voting matrix [7] for urban-area detection. Moreover, for the voting matrix formulation, we have introduced an improved orientation-sensitive technique using the 2-D elliptical Gaussian function, which exploits the orientation information of the feature points. Altogether, our proposed contributions increased the detection accuracy of ur- ban areas by $17 \%$ on the used data set. Our further plans include more improvement (Otsu's thresholding step may result in false detections if no urban area is presented in the image) and solving complex problems (e.g., extraction of building contours), where the introduced method will be used as a preprocessing step for decreasing the field of interest and presenting valuable information about the given area.

\section{ACKNOWLEDGMENT}

The authors would like to thank to B. Sirmaçek (author of [7]) for evaluating her method on the given data set for comparison. They would also like to thank A. Ondok and B. Manno for labeling the ground-truth data used in the evaluation process, and the anonymous reviewers and the Associate Editor for their valuable comments. Test images were provided by the Hungarian Institute of Geodesy, Cartography, and Remote Sensing (FÖMI).

\section{REFERENCES}

[1] J.A. Benediktsson, M. Pesaresi, and K. Arnason, "Classification and feature extraction for remote sensing images from urban areas based on morphological transformations," IEEE Trans. Geosci. Remote Sens., vol. 41, no. 9, pp. 1940-1949, Sep. 2003.

[2] P. Zhong and R. Wang, "A multiple conditional random fields ensemble model for urban area detection in remote sensing optical images," IEEE Trans. Geosci. Remote Sens., vol. 45, no. 12, pp. 3978-3988, Dec. 2007.

[3] L. Martinez-Fonte, S. Gautama, W. Philips, and W. Goeman, "Evaluating corner detectors for the extraction of man-made structures in urban areas," in Proc. IEEE Int. Geosci. Remote Sens. Symp., 2005, pp. 237-240.

[4] C. Harris and M. Stephens, "A combined corner and edge detector," in Proc. 4th Alvey Vis. Conf., 1988, pp. 147-151.

[5] S. M. Smith and J. M. Brady, "SUSAN-A new approach to low level image processing," Int. J. Comput. Vis., vol. 23 , no. 1, pp. 45-78, May 1997.

[6] B. Sirmaçek and C. Ünsalan, "Urban-area and building detection using SIFT keypoints and graph theory," IEEE Trans. Geosci. Remote Sens., vol. 47, no. 4, pp. 1156-1167, Apr. 2009.

[7] B. Sirmaçek and C. Ünsalan, "Urban area detection using local feature points and spatial voting," IEEE Geosci. Remote Sens. Lett., vol. 7, no. 1, pp. 146-150, Jan. 2010.

[8] A. Kovács and T. Szirányi, "Harris function based active contour external force for image segmentation," Pattern Recognit. Lett., vol. 33, no. 9, pp. 1180-1187, Jul. 2012.

[9] N. Otsu, "A threshold selection method from gray-level histograms," IEEE Trans. Syst., Man, Cybern., vol. SMC-9, no. 1, pp. 62-66, Jan. 1979.

[10] V. Kyrki and J. K. Kamarainen, "Simple Gabor feature space for invariant object recognition," Pattern Recognit. Lett., vol. 25, no. 3, pp. 311-318, Feb. 2004.

[11] S. Kumar and M. Hebert, "Man-made structure detection in natural images using a causal multiscale random field," in Proc. IEEE Conf. Comput. Vis. Pattern Recog., 2003, pp. 119-126.

[12] E. Rosten, R. Porter, and T. Drummond, "FASTER and better: A machine learning approach to corner detection," IEEE Trans. Pattern Anal. Mach. Intell., vol. 32, no. 1, pp. 105-119, Jan. 2010.

[13] T. Lindeberg, "Feature detection with automatic scale selection," Int. J. Comput. Vis., vol. 30, no. 2, pp. 77-116, Nov. 1998.

[14] D. G. Lowe, "Distinctive image features from scale-invariant keypoints," Int. J. Comput. Vis., vol. 60, no. 2, pp. 91-110, Nov. 2004.

[15] C. Benedek and T. Sziranyi, "Change detection in optical aerial images by a multi-layer conditional mixed Markov model," IEEE Trans. Geosci. Remote Sens., vol. 47, no. 10, pp. 3416-3430, Oct. 2009.

[16] K. Mikolajczyk and C. Schmid, "Scale and affine invariant interest point detectors," Int. J. Comput. Vis., vol. 60, no. 1, pp. 63-86, Oct. 2004.

[17] B. Sirmaçek and C. Ünsalan, "A probabilistic framework to detect buildings in aerial and satellite images," IEEE Trans. Geosci. Remote Sens., vol. 49, no. 1, pp. 211-221, Jan. 2011. 


\title{
Improved Harris Feature Point Set for Orientation-Sensitive Urban-Area Detection in Aerial Images
}

\author{
Andrea Kovács, Student Member, IEEE, and Tamás Szirányi, Senior Member, IEEE
}

\begin{abstract}
This letter addresses the automatic detection of urban area in remotely sensed images. As manual administration is time consuming and unfeasible, researchers have to focus on automated processing techniques, which can handle various image characteristics and huge amount of data. The applied method extracts feature points in the first step, which is followed by the construction of a voting map to represent urban areas. Finally, an adaptive decision making is performed to find urban areas. This letter presents methodological contributions in two key issues to the algorithm: 1) An automatically extracted Harris-based feature point set is introduced for the first step, which is able to represent urban areas more precisely. 2) An improved orientation-sensitive voting technique is proposed, exploiting the orientation information calculated in the local neighborhood of points. Evaluation results show that the proposed contributions increase the detection accuracy of urban areas.
\end{abstract}

Index Terms-Aerial images, modified Harris detector, orientation sensitivity, spatial voting, urban-area detection.

\section{INTRODUCTION}

$\mathbf{T}$ HE automatic detection of urban areas in optical aerial images means a great support in urban development analysis, map updating, and disaster management, and also helps municipalities in long-term residential area planning. Unfortunately, the images may cover large areas, and they can be taken in altering weather and illumination conditions, causing very different image features. Moreover, as urban areas are usually dynamically changing, continuous administration is required to have up-to-date information. Since manual administration is time consuming and unfeasible, researchers have to focus on automated processing techniques, which can handle various image characteristics and huge amount of data.

A wide range of automatic urban (a.k.a. built-in) area detection techniques have been introduced in recent years. The first group of such methods needs specific training data to detect urban areas, such as [1], using a differential morphological profile to record structural image information then applied feature extraction and neural network for classifying the fea-

Manuscript received May 25, 2012; revised September 14, 2012; accepted October 8, 2012. This work was supported by the Hungarian Scientific Research Fund under Grant 76159 and Grant 80352.

The authors are with the Distributed Events Analysis Research Laboratory, Computer and Automation Research Institute, Hungarian Academy of Sciences, 1111 Budapest, Hungary, and also with the Faculty of Information Technology, Pazmany Peter Catholic University, 1083 Budapest, Hungary (e-mail: andrea.kovacs@sztaki.mta.hu; sziranyi@ sztaki.mta.hu).

Color versions of one or more of the figures in this paper are available online at http://ieeexplore.ieee.org.

Digital Object Identifier 10.1109/LGRS.2012.2224315 tures, and [2], presenting a multiple conditional random-field ensemble model to incorporate multiple features and learn their contextual information.

Our letter followed another methodology, i.e., to construct a direct method that does not need any training data for urban-area extraction. Martinez-Fonte et al. [3] showed that corner detectors (Harris [4] and SUSAN [5]) are efficient tools for distinguishing different types of structures (manmade versus natural structures) present in the image. Since then, other works also applied interest point detectors for urbanarea detection; [6] used scale-invariant feature transform (SIFT) integrated with graph theory. The results were promising, but the computational complexity and time were quite significant. To reduce computational requirements, the same authors introduced a novel technique using Gabor feature points and spatial voting [7].

In this letter, we first introduce a new automatically extracted feature point set, which is called Modified Harris for Edges and Corners (MHEC) for urban-area detection, which was applied for effective object contour detection in our earlier work [8]. As the density of extracted feature points is higher in the residential areas [3], building a probability map based on this feature point set can identify urban areas. After having a local feature point set, we apply the voting matrix strategy of [7] to get a probability map of the urban area. To improve the accuracy of this step, we also propose a novel orientationsensitive technique for constructing the voting matrix. Finally, urban areas are calculated by a decision making step. Fig. 1 shows the main algorithmic steps of the method. In the experimental part, we will show that the introduced method is able to outperform other interest point detectors; moreover, the proposed orientation-sensitive voting matrix is also able to improve the performance of the previously introduced Gaborbased algorithm [7].

\section{MODIFIED HARRIS FOR EDGES AND CORNERS}

We introduce a novel MHEC feature point set for urbanarea detection, which is based on the Harris corner detector [4] but adopts a modified characteristic function. The advantage of the improved detector is that it is automatic and it is able to recognize not just corners but edges as well. Therefore, it gives an efficient tool for characterizing contour-rich regions, such as urban areas. The improved characteristic function was also successfully applied earlier for object contour recognition with parametric active contour algorithms [8].

By considering the behavior of the Harris matrix and its eigenvalues (denoted by $\lambda_{1}$ and $\lambda_{2}$ ), we proposed the following 


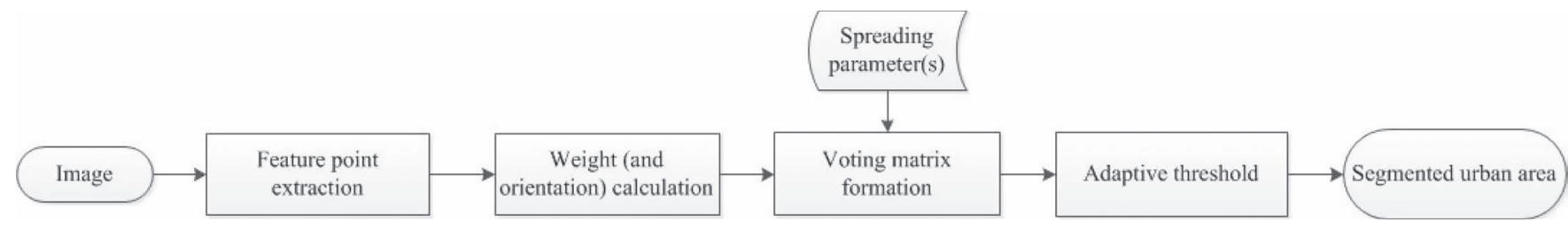

Fig. 1. Simplified diagram of the workflow.

modification of the $R$ characteristic function in our earlier work [8]:

$$
R_{\text {mod }}=\max \left(\lambda_{1}, \lambda_{2}\right)
$$

As our aim is to emphasize edge and corner regions simultaneously, we can exploit the fact that they both have one large eigenvalue. Therefore, the suggested $R_{\text {mod }}$ value is able to separate homogeneous and nonhomogeneous regions of the image.

Feature points are calculated as the local maxima of $R_{\text {mod }}$. A pixel $p_{i}=\left(x_{i}, y_{i}\right)$ is the element of the $P$ feature point set, if it has the largest $R_{\text {mod }}\left(p_{i}\right)$ value compared with its neighbors in a surrounding $b_{i}=\left\{\left[x_{i}-1, x_{i}+1\right] \times\left[y_{i}-1, y_{i}+1\right]\right\}$ window and its $R_{\bmod }\left(p_{i}\right)$ value exceeds a given $T_{\max }$ threshold, i.e.,

$P=\left\{p_{i}: R_{\text {mod }}\left(p_{i}\right)>T_{\max } \operatorname{AND} p_{i}=\arg \max _{r \in b_{i}} R_{\bmod }(r)\right\}$.

Here, the $T_{\max }$ threshold is calculated by Otsu's method [9] for each image adaptively; therefore, it can handle different image characteristics.

By investigating the smaller eigenvalue $\min \left(\lambda_{1}, \lambda_{2}\right)$, edge and corner points can be separated. If $\min \left(\lambda_{1}, \lambda_{2}\right)$ is over an adaptive $T_{\min }$ threshold (also calculated by Otsu's method), then the point is a corner point candidate; otherwise, it is an edge point. The separation of edge and corner points will be used in Section III-B.

The proposed feature point detector is completely automatic and does not need any parameter tuning, unlike the referred Gabor mechanism [7]. The method is also not sensitive to the $g$ scale parameter of the Gaussian smoothing used in the Harris corner detection; we picked the $g=0.5$ value for every test case, regardless of resolution or any other image feature. Therefore, we did not present it in Fig. 1 as the input parameter. Additionally, the aforementioned $T_{\min }$ and $T_{\max }$ thresholds are defined with Otsu's adaptive method.

In the next step (see Fig. 1), we calculate a weight value (presented in [7]) for each feature point that represents the strength of the point's neighborhood. Following [7], we binarize the $R_{\text {mod }}$ map with the adaptive $T_{\max }$ threshold to get a $B$ binary map. In $B$, pixels of $R_{\bmod }$ over $T_{\max }$ get 1 ; others get 0 value. By definition, every member of the $P$ feature point set will have a value of 1 [see (2)]. Let $w_{i}$ denote the weight of the $p_{i}$ point, which is calculated as the natural logarithm of the number of pixels connected to $p_{i}$ in the $B$ binary map. Two pixels are connected in $B$ if both pixels are 1, and a path of pixels with a value of 1 exists between them. If the number of connected pixels is less than 10 (defined based on the recommendation of [7]), the point is classified as nonsignificant and eliminated from the feature point set. Points in larger sections, like roads, may have higher weights than others, resulting in an unbalanced weighting function;
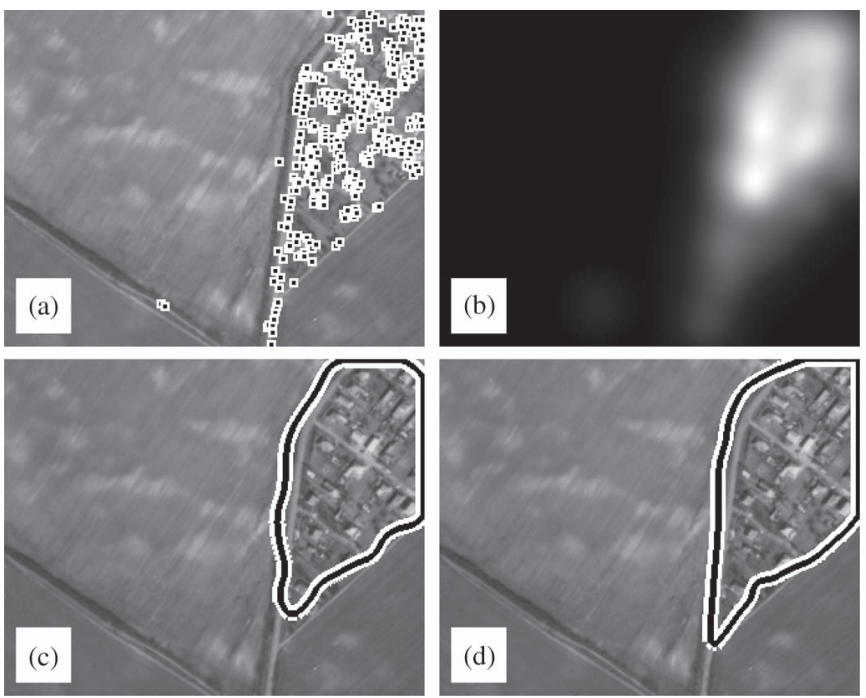

Fig. 2. Steps of the urban-area extraction for the Szada 1 image with the proposed MHEC feature point set. (a) Extracted feature point set. (b) Voting matrix of the referred nonoriented process [7]. (c) Detected urban area applying the nonoriented process. (d) Detected urban area applying the improved orientation-sensitive process.

therefore, the reason for applying the natural logarithm function on the calculated weights is to get steady $w_{i}$ values.

The modified Harris point set for the $\mathrm{Szada}_{1}$ image is shown in Fig. 2(a). The modified (extended) point set has members in edge regions (like roads) as well, and points are densely located in the built-in area. Only a few points are situated in the nonurban area.

\section{Orientation-SEnsitive Urban-AREA EXtraction}

\section{A. Orientation-Sensitive Voting Matrix Formation}

Sirmaçek and Ünsalan [7] assumes that, around Gabor feature points [10], an urban area can be found with a high probability. Thus, it defines a Gaussian domain around each $p_{i}$ point with $\sigma_{i}$ standard deviation, where the highest vote is the point, and according to the spatial distance, the vote is decreasing. $\sigma_{i}$ controls the spatial effect of a point; if it is higher, a point has wider impact. The significance of a point is proportional to its weight; thus, $\sigma_{i}$ is estimated by $w_{i}$. The $V$ voting matrix for the $(x, y)$ pixel is defined as

$$
V(x, y)=\sum_{i=1}^{K} \frac{1}{2 \pi \sigma_{i}^{2}} e^{-\frac{\left(x-x_{i}\right)^{2}+\left(y-y_{i}\right)^{2}}{2 \sigma_{i}^{2}}}
$$

where $K$ is the total number of feature points, and $\sigma_{i}=5 \times w_{i}$. Fig. 2(b) shows the generated $V$ map for the $\mathrm{Szada}_{1}$ image. Finally, the urban area is defined by an adaptive decision making step. Otsu's thresholding technique [9] is applied on $V$ to distinguish the urban area from the background. The detection result is shown in Fig. 2(c). 

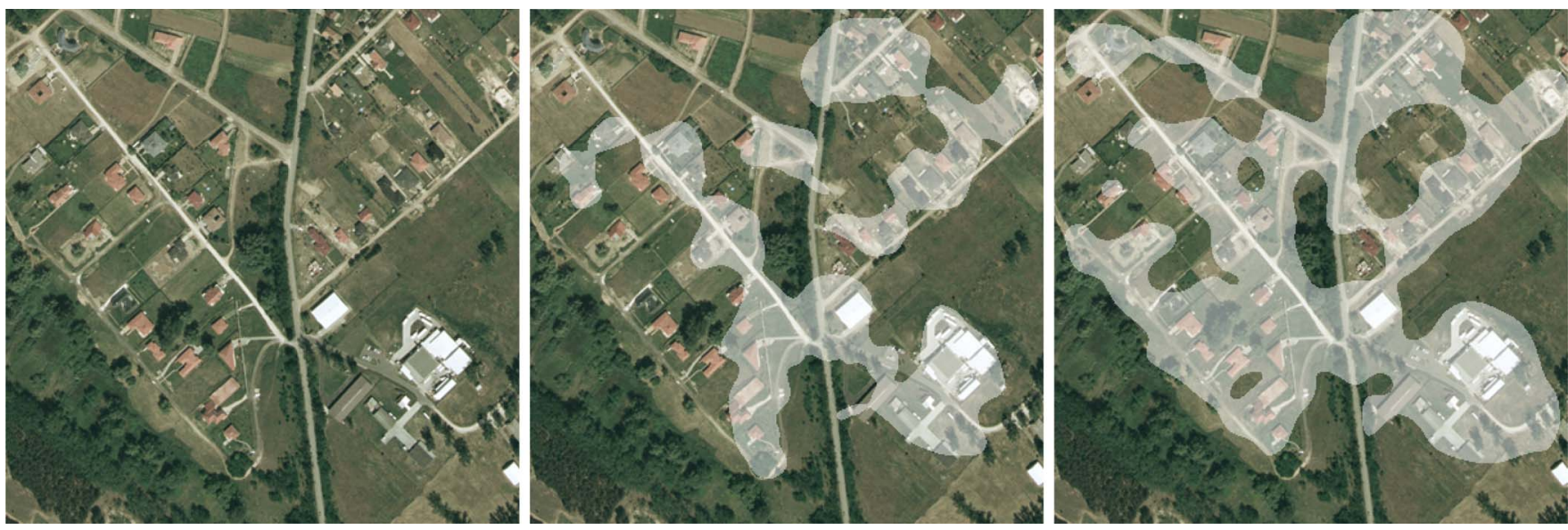

Fig. 3. Detection results for the Szada 5 image with different voting matrix formation techniques. (Left) Original image. (Middle) Result of the original nonoriented method [7] ( $F$-measure value: 0.548$)$. (Right) Result of the proposed orientation-sensitive method ( $F$-measure value: 0.717$)$.

The disadvantage of the method is that it is not able to detect straight lines accurately because of the circular Gaussian representation. If a straight urban-area border (like a road segment or a row of houses) is represented by sparse feature points, then the voting matrix will have high values only in the neighborhood of the points; therefore, the detection will result in a less accurate wavy urban-area border. Moreover, high curvature boundaries are also inaccurately detected.

To compensate these drawbacks, we introduce the orientation-sensitive extension of the voting matrix. Our idea is to use the direction information of Gabor filters [10] when constructing the voting matrix. Therefore, we propose to apply the 2-D elliptical Gaussian function at the feature point $\left(x_{i}, y_{i}\right)$, which can model orientation:

$$
\begin{aligned}
& G_{i}(x, y)=A_{i} \cdot E_{i}(x, y) \\
& E_{i}(x, y)=e^{-\left(a_{i}\left(x-x_{i}\right)^{2}+2 b_{i}\left(x-x_{i}\right)\left(y-y_{i}\right)+c_{i}\left(y-y_{i}\right)^{2}\right)}
\end{aligned}
$$

where the calculation of $A_{i}$ is given in (7), and $a_{i}, b_{i}$, and $c_{i}$ are calculated in the following way:

$$
\begin{aligned}
a_{i} & =\frac{\cos ^{2} \theta}{2 \sigma_{i, x}^{2}}+\frac{\sin ^{2} \theta}{2 \sigma_{i, y}^{2}} \\
b_{i} & =-\frac{\sin 2 \theta}{4 \sigma_{i, x}^{2}}+\frac{\sin 2 \theta}{4 \sigma_{i, y}^{2}} \\
c_{i} & =\frac{\sin ^{2} \theta}{2 \sigma_{i, x}^{2}}+\frac{\cos ^{2} \theta}{2 \sigma_{i, y}^{2}}
\end{aligned}
$$

where $\sigma_{i, x}$ and $\sigma_{i, y}$ are the spreads of the blob in the $x$ - and $y$ directions, and $\theta$ is the orientation for the $\left(x_{i}, y_{i}\right)$ feature point.

This interpretation represents a 2-D elliptical Gaussian function, which is rotated by a clockwise angle $\theta$. If we define $\theta$ as the orientation of the Gabor filter, which extracted the feature point, then we can exploit the additional direction information in the voting matrix formulation by expanding a point's impact in the main $\theta$-direction.

Regarding this consideration, we define the following oriented voting matrix:

$$
V_{\theta}(x, y)=\sum_{i=1}^{K} G_{i}(x, y)=\sum_{i=1}^{K} \frac{1}{2 \pi\left(\sigma_{i, x}^{2}+\sigma_{i, y}^{2}\right)} \cdot E_{i}(x, y) .
$$

We set the $\sigma_{i, y} / \sigma_{i, x}=2$ ratio to avoid too moderate voting in the minor axis direction, resulting in false negative hits in the detection step.

The effect of the orientation sensitivity is shown in Fig. 3, where the middle image shows the result of the original nonoriented method for Gabor points [7], while the right image shows the result of the improved orientation-sensitive technique $\left(\sigma_{i, x}=3 \times w_{i}\right.$ and $\left.\sigma_{i, y}=6 \times w_{i}\right)$. To quantitatively compare the results, the gained $F$-measure scores are also presented. (For further evaluation details see Section IV.)

\section{B. Orientation-Sensitive Voting Matrix Formation for the Novel Feature Point Set}

In case of a Gabor feature point set, defining a point's orientation does not need any extra computation; therefore, it is favorable to exploit the additional information. However, there may be other interest point detectors where the orientation has to be calculated in a distinct step. Moreover, in some cases, no such direction can be interpreted for points without further consideration. For example, when using the Harris corner detector, more than one main direction can be assigned to a point, which may pose many interpretation questions. (How is it to decide if a direction is significant? How many significant directions have to be calculated?)

In our case, the feature point set proposed in Section II will consist of both edge and corner points. Thus, calculating multiple directions featuring corner points is problematic. Meanwhile, edge points represent a main orientation, which can be defined, for example, by analyzing the small neighborhood, as in [11]. Therefore, we propose to apply a mixture of voting matrices $\left(V\right.$ and $\left.V_{\theta}\right)$. After dividing the feature point set into two subsets, i.e., corner and edge points (as described in Section II), the original circular Gaussian field $[V$ in (3)] is calculated for corner points, and the improved orientationsensitive elliptical field $\left[V_{\theta}\right.$ in (7)] is calculated for edge points. The final voting matrix is then given as the pixelwise sum of the two precalculated matrices. Fig. 2(d) shows the result of the joint detection technique for the proposed MHEC point set.

\section{EXPERIMENTS}

For testing the performance of the proposed feature point set and the improved orientation-sensitive voting matrix, we 
used the Szada data set provided by the Hungarian Institute of Geodesy, Cartography, and Remote Sensing (FÖMI; earlier used in [15]). The Szada test set contains 11 aerial images taken by FÖMI in 1984, 2000, and 2005, showing the Szada village and its close proximity. Our aerial images have a different spatial resolution, i.e., 1.5 or $0.5 \mathrm{~m} / \mathrm{pixel}$; images are either grayscale or colored. The size of the images is varying from $320 \times 256$ to $996 \times 558$ pixels. Therefore, the data set contains diverse images to represent different urban region characteristics and to show the robustness of the proposed techniques.

In the first part of the evaluation process we investigate the efficiency of different interest point detectors for urban-area detection with the original nonoriented voting matrix technique. In the second part, we test the performance of the improved orientation-sensitive voting matrix for selected feature point detectors.

For comparing the results, we use $P$ precision, $R$ recall, and $F$-measure values, i.e.,

$$
P=\frac{\mathrm{TP}}{\mathrm{TP}+\mathrm{FP}} \quad R=\frac{\mathrm{TP}}{\mathrm{TP}+\mathrm{FN}} \quad F=2 \cdot \frac{P \cdot R}{P+R}
$$

where TP, FP, and FN denote the number of true positive, false positive, and false negative detected pixels, respectively.

When defining an urban area, various interpretations might be considered.

- Are roads and gardens parts of the urban area?

- Should buildings be separately or collectively marked?

To handle these subjective cases, we asked three individuals to label the urban area manually. If a pixel was labeled as urban by at least two persons, then it was treated as a part of a built-in area in the ground-truth-based evaluation process.

\section{A. Tests on Different Interest Point Detectors}

To evaluate the performance of our proposed feature point detector, we tested the original voting matrix technique for different point detectors. Since there exist a large number of point detection methods [16], we picked detectors so as to cover a wide range of detection techniques. Harris [4] and SUSAN [5] were proposed in [3] for extraction of manmade structures; previous works of Sirmaçek and Ünsalan used Lowe's SIFT [14] method in [6], Gabor filtering [10] in [7], and Features from Accelerated Segment Test (FAST) [12] and Lindeberg's blob detector Laplacian of Gaussian (LoG) [13] in [17]. Thus, we chose these detectors for evaluation and comparison.

Table I shows the calculated average $F$-measure values [see (8)] for different interest point detection methods. Our proposed MHEC method outperforms the other detectors and achieves more than 0.8 for a mean $F$-measure value.

The computation time was calculated for the MHEC feature point extraction step on the Szada 1 test image (with $320 \times$ 256 size), and altogether, $0.6 \mathrm{~s}$ has been achieved on a personal computer with an Intel Core i7 2.67-GHz central processing unit with 4-GB random access memory and MATLAB R2011b. This is a fairly good time compared with [7], where Gabor filtering and local feature point extraction steps needed approximately $1.5 \mathrm{~s}$ for an image with a similar size $(235 \times 265)$.
TABLE I

Average $F$-Measure Score (MeAn \pm Standard Deviation) For the Evaluated Feature Point Detector Methods FOR THE SZADA DATA SET

\begin{tabular}{|c|c|}
\hline Feature point detector & Average F-measure Score \\
\hline MHEC (proposed) & $\mathbf{0 . 8 0 1} \pm \mathbf{0 . 0 4 4}$ \\
\hline FAST [12] & $0.792 \pm 0.072$ \\
\hline SUSAN [5] & $0.778 \pm 0.084$ \\
\hline LoG [13] & $0.750 \pm 0.061$ \\
\hline SIFT [14] & $0.670 \pm 0.093$ \\
\hline Harris [4] & $0.667 \pm 0.154$ \\
\hline Gabor [7] & $0.646 \pm 0.124$ \\
\hline
\end{tabular}

\section{B. Tests on Orientation Sensitivity}

To test the orientation sensitivity, we first chose interest point detectors, where the orientation is reasonable. As mentioned in Section III-B, this additional information cannot be interpreted for every detector (like Harris detector or blob detectors) and sometimes needs more consideration like MHEC).

For the originally used Gabor points, orientation information for a point is given when determining the direction of the Gabor filter. Therefore, exploiting orientation information does not need any additional computation. Furthermore, detectors based on edge-enhancing techniques extract edge points as well, which can be thus used for orientation-sensitive urbanarea detection with further considerations.

In our evaluation step, we picked the proposed MHEC method and the SUSAN detector to show the performance of the orientation-sensitive voting matrix. In both cases, we used the mixture of nonoriented and oriented voting matrix techniques (see Section III-B), as edge and corner points can be separated. The separation procedure for the MHEC method was described in Section II. For the SUSAN method, the main principle can be used for both edge and corner detection purposes. Depending on the value of the geometric threshold, the algorithm is able to find edge or corner points (see [5] for further details); therefore, it can be used to distinguish edges and corners.

Fig. 4 shows the comparison of the performance for the three selected algorithms. The light bar is the original $F$-measure performance value achieved by the referred nonoriented method, while the dark one is the result achieved by the improved orientation-sensitive voting technique.

Spreading the parameter values of the orientation-sensitive voting matrix, $\sigma_{i, x}$ and $\sigma_{i, y}$ were selected based on the number of detected points and on the distribution of weight values. While the $\sigma_{i, y} / \sigma_{i, x}$ ratio handles the orientation sensitivity, i.e., the shape of the effect (depending on the number of points), $\sigma_{i, x}$ and $\sigma_{i, y}$ values are responsible for the coverage of a point (depending on the variance of weight values).

In case of Gabor points, we applied $\sigma_{i, x}=3 \times w_{i}$ and $\sigma_{i, y}=$ $6 \times w_{i}$ values. As this detector gives the highest number of points and the weight values have a large variance, orientation and saliency are represented by effectively by the points.

In case of SUSAN, the oriented $\sigma_{i, x}, \sigma_{i, y}$ (for edge points) and nonoriented $\sigma_{i}$ (for corner points) values were manually tuned with the following restrictions: $\sigma_{i, y} / \sigma_{i, x}=2$ ratio 


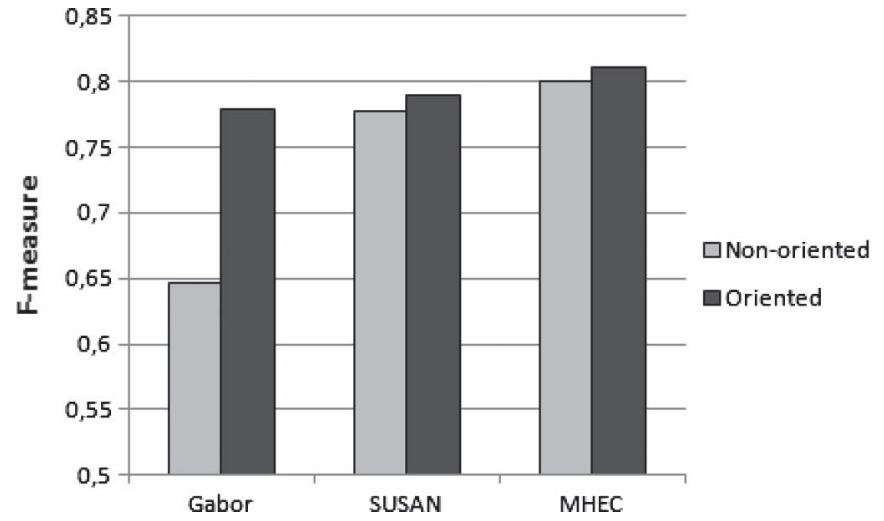

Fig. 4. Detection results based for different voting matrix techniques. (Left) Original nonoriented method [7]. (Right) Proposed orientation-sensitive method.

and $\sigma_{i, x}<\sigma_{i}<\sigma_{i, y}$ (a typical spreading parameter setting was $\sigma_{i, x}=8 \times w_{i}, \sigma_{i, y}=16 \times w_{i}$, and $\sigma_{i}=14 \times w_{i}$ used for $\mathrm{Szada}_{1}$ in Fig. 2). These considerations were empirically defined to balance the effect of corner and edge points. The variance of the weights was much lower than in the case of the Gabor detector; therefore, we selected larger multipliers in the case of $\sigma_{i, x}$ and $\sigma_{i, y}$ to exaggerate the saliency effect. The exact parameter values also depended on the resolution of the image; the higher the resolution, the wider spatial effect for a point has to be selected. Therefore, in the case of images with $0.5 \mathrm{~m} /$ pixel resolution, a typical parameter setting $\sigma_{i, x}=12 \times$ $w_{i}, \sigma_{i, y}=24 \times w_{i}$, and $\sigma_{i}=20 \times w_{i}$ was applied.

In the case of MHEC, the behavior of the detector was similar to the Gabor case, but the number of points was lower. Therefore, we set the $\sigma_{i, y} / \sigma_{i, x}$ ratio higher; to represent the orientation sensitivity more efficiently, we applied $\sigma_{i, x}=$ $2 \times w_{i}, \sigma_{i, y}=6 \times w_{i}$, and $\sigma_{i}=3 \times w_{i}$ for lower resolution, and $\sigma_{i, x}=2 \times w_{i}, \sigma_{i, y}=8 \times w_{i}$ and $\sigma_{i}=6 \times w_{i}$ for higher resolution. As the variance of the weight values was high, the multipliers of the $w_{i}$ weight values are smaller than in the case of SUSAN.

According to Fig. 4, the orientation-sensitive representation was able to improve the performance of urban-area detection. The improvement was the most significant for Gabor points, where no additional calculation was needed. Moreover, it caused a slight increase in the performances of SUSAN and MHEC as well.

\section{CONCLUSION}

We have proposed a feature point set based on the modification of the Harris corner detection method for urban-area extraction in aerial images. The point extraction method is fully automatic and is able to emphasize edge and corner points in the image equally; therefore, it serves as a suitable base for the construction of the voting matrix [7] for urban-area detection. Moreover, for the voting matrix formulation, we have introduced an improved orientation-sensitive technique using the 2-D elliptical Gaussian function, which exploits the orientation information of the feature points. Altogether, our proposed contributions increased the detection accuracy of ur- ban areas by $17 \%$ on the used data set. Our further plans include more improvement (Otsu's thresholding step may result in false detections if no urban area is presented in the image) and solving complex problems (e.g., extraction of building contours), where the introduced method will be used as a preprocessing step for decreasing the field of interest and presenting valuable information about the given area.

\section{ACKNOWLEDGMENT}

The authors would like to thank to B. Sirmaçek (author of [7]) for evaluating her method on the given data set for comparison. They would also like to thank A. Ondok and B. Manno for labeling the ground-truth data used in the evaluation process, and the anonymous reviewers and the Associate Editor for their valuable comments. Test images were provided by the Hungarian Institute of Geodesy, Cartography, and Remote Sensing (FÖMI).

\section{REFERENCES}

[1] J.A. Benediktsson, M. Pesaresi, and K. Arnason, "Classification and feature extraction for remote sensing images from urban areas based on morphological transformations," IEEE Trans. Geosci. Remote Sens., vol. 41, no. 9, pp. 1940-1949, Sep. 2003.

[2] P. Zhong and R. Wang, "A multiple conditional random fields ensemble model for urban area detection in remote sensing optical images," IEEE Trans. Geosci. Remote Sens., vol. 45, no. 12, pp. 3978-3988, Dec. 2007.

[3] L. Martinez-Fonte, S. Gautama, W. Philips, and W. Goeman, "Evaluating corner detectors for the extraction of man-made structures in urban areas," in Proc. IEEE Int. Geosci. Remote Sens. Symp., 2005, pp. 237-240.

[4] C. Harris and M. Stephens, "A combined corner and edge detector," in Proc. 4th Alvey Vis. Conf., 1988, pp. 147-151.

[5] S. M. Smith and J. M. Brady, "SUSAN-A new approach to low level image processing," Int. J. Comput. Vis., vol. 23 , no. 1, pp. 45-78, May 1997.

[6] B. Sirmaçek and C. Ünsalan, "Urban-area and building detection using SIFT keypoints and graph theory," IEEE Trans. Geosci. Remote Sens., vol. 47, no. 4, pp. 1156-1167, Apr. 2009.

[7] B. Sirmaçek and C. Ünsalan, "Urban area detection using local feature points and spatial voting," IEEE Geosci. Remote Sens. Lett., vol. 7, no. 1, pp. 146-150, Jan. 2010.

[8] A. Kovács and T. Szirányi, "Harris function based active contour external force for image segmentation," Pattern Recognit. Lett., vol. 33, no. 9, pp. 1180-1187, Jul. 2012.

[9] N. Otsu, "A threshold selection method from gray-level histograms," IEEE Trans. Syst., Man, Cybern., vol. SMC-9, no. 1, pp. 62-66, Jan. 1979.

[10] V. Kyrki and J. K. Kamarainen, "Simple Gabor feature space for invariant object recognition," Pattern Recognit. Lett., vol. 25, no. 3, pp. 311-318, Feb. 2004.

[11] S. Kumar and M. Hebert, "Man-made structure detection in natural images using a causal multiscale random field," in Proc. IEEE Conf. Comput. Vis. Pattern Recog., 2003, pp. 119-126.

[12] E. Rosten, R. Porter, and T. Drummond, "FASTER and better: A machine learning approach to corner detection," IEEE Trans. Pattern Anal. Mach. Intell., vol. 32, no. 1, pp. 105-119, Jan. 2010.

[13] T. Lindeberg, "Feature detection with automatic scale selection," Int. J. Comput. Vis., vol. 30, no. 2, pp. 77-116, Nov. 1998.

[14] D. G. Lowe, "Distinctive image features from scale-invariant keypoints," Int. J. Comput. Vis., vol. 60, no. 2, pp. 91-110, Nov. 2004.

[15] C. Benedek and T. Sziranyi, "Change detection in optical aerial images by a multi-layer conditional mixed Markov model," IEEE Trans. Geosci. Remote Sens., vol. 47, no. 10, pp. 3416-3430, Oct. 2009.

[16] K. Mikolajczyk and C. Schmid, "Scale and affine invariant interest point detectors," Int. J. Comput. Vis., vol. 60, no. 1, pp. 63-86, Oct. 2004.

[17] B. Sirmaçek and C. Ünsalan, "A probabilistic framework to detect buildings in aerial and satellite images," IEEE Trans. Geosci. Remote Sens., vol. 49, no. 1, pp. 211-221, Jan. 2011. 